\title{
A Case of PMM2-CDG Caused by an A108V Mutation Associated With a Heterozygous 70 Kilobases Deletion
}

Elodie Lebredonchel ( $\nabla$ elodie.lebredonchel@chu-lille.fr)

UGSF: Unite de Glycobiologie Structurale et Fonctionnelle https://orcid.org/0000-0001-5119-6005

\section{A. Riquet}

CHU Lille Pôle Enfant: Centre Hospitalier Universitaire de Lille Pole Enfant

D. Neut

$\mathrm{CH}$ Boulogne sur Mer: Centre Hospitalier de Boulogne sur Mer

F. Broly

Centre Hospitalier Régional Universitaire de Lille Centre de Biologie Pathologie: Centre Hospitalier

Regional Universitaire de Lille Pole de Biologie Pathologie Genetique

\section{G. Matthijs}

KU Leuven Center for Human Genetics: Katholieke Universiteit Leuven Centrum Menselijke Erfelijkheid

\section{A. Klein}

CHRU de Lille CBP: Centre Hospitalier Regional Universitaire de Lille Pole de Biologie Pathologie

Genetique

\section{F. Foulquier}

UGSF: Unite de Glycobiologie Structurale et Fonctionnelle

\section{Research Article}

Keywords: $\mathrm{CDG}$, PMM2, deletion mutation, PMM2 CDG, whole genome sequencing

Posted Date: February 1st, 2022

DOI: https://doi.org/10.21203/rs.3.rs-1231444/v1

License: (c) (i) This work is licensed under a Creative Commons Attribution 4.0 International License. Read Full License 


\section{Abstract}

Background: Congenital Disorders of Glycosylation (CDG) are a large group of inherited inborn errors of metabolism with more than 140 different CDG types reported to date (1). The first characterized is also the most common, PMM2-CDG, with an autosomal recessive transmission. The PMM2 gene is encoding a phosphomannomutase.

Case report: We report the case of a French child, from healthy and unrelated parents, presenting congenital ataxia with hypotonia, hyperlaxity, inverted nipples and alteration of the liver function and coagulation parameters. Transferrin isoelectrofocusing revealed a typical type I CDG profile. Direct Sanger sequencing and quantitative PCR of PMM2 revealed a unique and novel genotype. On one allele, the patient was heterozygote with a known missense variant rs200203569

NM_000303.3(PMM2):c.323C>T, p.Ala108Val in exon 4. On the second allele, WGS confirmed the presence of a novel heterozygous 70453 bp deletion encompassing 6 exons of PMM2 at position chr16:8,897,826-8,968,278.

Conclusion: As the maximum length of a PMM2 deletion reported by HGMS database is $28 \mathrm{~kb}$, we identified the largest heterozygous deletion reported so far. The observation reveals the impact of a thorough diagnostic testing on genetic counselling: hasty conclusion of homozygosity in the case of a relatively rare variant found in an index patient with unrelated parents can be avoided using whole genome sequencing.

\section{Introduction}

Congenital Disorders of Glycosylation (CDG) are a rapidly expanding family of genetic diseases. Today, 140 different CDG subtypes have been reported (1) categorized in 2 groups: CDG-I, affecting steps before the oligosaccharide precursor transfer in the endoplasmic reticulum, and CDG-II, affecting the steps following the transfer, mostly in the Golgi apparatus. The first patient cases were reported 40 years ago by Jaeken et al., (2). Mutations in PMM2 (OMIM 601785), a gene on chromosome 16p13 encoding a phosphomannomutase. This enzyme catalyzes the conversion in the cytosol of mannose6-P to mannose-1-P, necessary for the synthesis of donor substrates for glycosylation, GDPmannose, and DolPmannose.

First named CDG-la then changed into PMM2-CDG in 2009 (3), the disease is thought to represent $70 \%$ of the total CDG cases, with an estimated incidence around 1:20 000 (4). The spectrum of clinical phenotypes and severity is broad and is characterized with mainly a psychomotor development impairment associated with cerebellar hypoplasia, hypotonia, dysmorphia, and coagulopathy (5). The lethality rate in the first 4 years of life is about 20\%. Beyond childhood, PMM2-CDG patients have a good life expectancy (6). The number of PMM2 mutations classified in HGMD (https://my.qiagendigitalinsights.com) is 142 , most of these, 113 , are missense mutations and the most frequent is p.Arg141His (R141H) (7). We report the particular genotype of a PMM2-CDG patient with a 
heterozygous p.Ala108Val (A108V) mutation on one allele and a first-described $>70 \mathrm{~kb}$-deletion on the other allele.

\section{Case Report}

We report the case of a PMM2-CDG French child of Caucasian descent. She was born at term with a length of $48 \mathrm{~cm}$ for $3.110 \mathrm{~kg}$, after uncomplicated pregnancy with vaginal delivery. The AGPAR score was 10 both at 1 minute and 5 minutes and the newborn screening results were normal. The parents are unrelated and the 6-years older brother is healthy. Parents reported abnormal abrupt movements of the child after birth that stopped spontaneously.

Biologically, elevated transaminases TGO $=50 \mathrm{UI} / \mathrm{L}, \mathrm{TGP}=51$ [normal values 10-35 UI/L] and abnormal coagulation parameters with ATIIIA $=63 \%$ [normal values $80-120 \%$ ] and FXI $53 \%$ at the lower limit [normal values $50-150 \%]$ were assayed. The cytology and the thyroid function were normal.

The clinical examination at 9 months of age revealed ataxia, hypotonia, hyperlaxity, strabismus, esotropia, feeding difficulties, and inverted nipples. The child was calm and exclusively breastfed with an absence of facial dysmorphia and no sleeping disorders. At that time, the girl presented an inability to reach a seated posture. The diagnosis of CDG was oriented by an abnormal pattern in serum transferrin isoelectrofocusing (8) with an elevation of asialo- and disialo-transferrin, typical from a type I CDG (Fig. 1). Brain MRI revealed cerebellar abnormalities with vermis hypoplasia. The child finally reaches a seated posture at 11 months of age.

\section{GENETIC TESTING}

Direct Sanger sequencing of the 8 exons of $P M M 2$ reported a seemingly homozygous variant rs200203569 NM_000303.3(PMM2): c.323C>T in exon 4. The variant leads to a missense substitution of alanine 108 to proline (p.Ala108Pro), commonly named A108V, that is known to be pathogenic (ClinVar, SIFT, Mutation Taster). The A108V mutation is quite rare and is often associated with $\mathrm{R} 141 \mathrm{H}$, the most common deleterious PMM2 mutation, in compound heterozygous patients (9). A homozygous presentation of $\mathrm{R} 141 \mathrm{H}$ variant is thought to be incompatible with life as no case was reported so far (10). For the A108V variant, the gnomAD (2.1) website reports a frequency of $0.0012 \%$ in the overall population. To our knowledge, no homozygous $\mathrm{A} 108 \mathrm{~V}$ patients are reported in the literature and, as the parents were unrelated, further genetic explorations were conducted. Direct Sanger sequencing of $P M M 2$ of the paternal DNA reported a heterozygous A108V mutation while no mutation was found in the mother. A quantitative PCR (qPCR) of the 13 exons was performed, showing a reduction of the DNA of the gene by $50 \%$ in the mother and the proband from exon 3 to exon 8 , the last exon of PMM2 (Fig. 2). A heterozygous deletion of $P M M 2$ gene was then suspected. To evaluate the extent of the deletion that goes beyond PMM2 gene, Whole Genome Sequencing (WGS) was performed. WGS was preferred to CGH array to accurately determine the exact position of the breakpoints. WGS allowed to delineate the deletion of 70453 bp in position chr16:8,897,826-8,968,278 (Fig. 3). In the HGMD database, the largest deletion reported is $28 \mathrm{~kb}$-long. 
The deletion also affects a part of CARHSP1 (Calcium Regulated Hear Stable Protein 1) (OMIM: 616885) gene that plays a role in TNF mRNA stabilization, seemingly not affecting the phenotype.

\section{Discussion And Conclusions}

In the present study, we described the case of a PMM2-CDG patient with congenital ataxia. The genotype identified in the child is novel. The clinical course was relatively mild for a child with PMM2-CDG as the child does not present facial dysmorphia. Given that the maternal mutation could not be detected upon Sanger sequencing, further investigation were performed to precise the genetic transmission of the disease.

A108V was first described in France (7) and its effect on the enzyme activity is unknown. When associated to a mutation in $\mathrm{R} 141 \mathrm{H}$ the remaining phosphomannomutase activity in leucocytes is $0.09 \%$ (11).

Quantitative PCR and WGS allowed to identify a large deletion on the maternal allele. A new deletion of 70453 bp in position chr16:8,897,826-8,968,278 could be accurately detected with WGS including 6 exons of PMM2 and a part of CARHSP1 gene. Knockout of CARHSP1 has demonstrated the role of CARHSP1 as a TNF-a mRNA stability enhancer (12). GnomAD database reports various loss of function heterozygote mutation for CARHSP1, indicating that the observed pathology is mainly due to the phosphomannomutase defect.

To our knowledge, this is the largest $P M M 2$ deletion reported so far. Our example illustrates the usefulness of WGS in the case of an apparent homozygous variant in an unrelated family. Wherever possible, compound heterozygosity has to be confirmed with a parental genetic study. As the disease transmission for the couple is $25 \%$, an antenatal diagnostic can now be proposed for future pregnancies.

\section{Abbreviations}

Congenital Disorder of Glycosylation (CDG), Phosphomannomutase 2 (PMM2), Whole Genome Sequencing (WGS), base pair (bp)

\section{Declarations}

\section{ETHICS APPROVAL AND CONSENT TO PARTICIPATE}

Informed consent was obtained from parents to proceed to a molecular diagnosis on the patient.

\section{CONSENT FOR PUBLICATION}

Informed consent was obtained from parents to publish data on the patient.

\section{AVAILABILITY OF DATA AND MATERIALS}


Any supplementatry data are available from the corresponding author on request.

\section{COMPETING INTERESTS}

The authors have declared that no competing interests exist.

\section{AUTHORS CONTRIBUTIONS}

Reported the case : N.D, R.A, Conducted the analyses : L.E, B.F, Analysed the data : L.E, B.F, Wrote the paper : L.E, FF, K.A, MG

\section{FUNDING}

This work was supported by the Agence Nationale de la Recherche (ANR: Solv-CDG).

\section{ACKNOWLEDGMENT}

We thank the patient's parents for their kind participation and support.

\section{References}

1. Ondruskova N, Cechova A, Hansikova H, Honzik T, Jaeken J. Congenital disorders of glycosylation: Still « hot » in 2020. Biochim Biophys Acta Gen Subj. janv 2021;1865(1):129751.

2. Jaeken J, Vanderschueren-Lodeweyckx M, Casaer P, Snoeck L, Corbeel L, Eggermont E, et al. Familial psychomotor retardation with markedly fluctuating serum prolactin, FSH and GH levels, partial TBGdeficiency, increased serum arylsulphatase A and increased CSF protein: a new syndrome?: 90. Pediatric Research. févr 1980;14(2):179-179.

3. Aebi M, Helenius A, Schenk B, Barone R, Fiumara A, Berger EG, et al. Carbohydrate-deficient glycoprotein syndromes become congenital disorders of glycosylation: an updated nomenclature for CDG. First International Workshop on CDGS. Glycoconj J. nov 1999;16(11):669-71.

4. Schollen E, Kjaergaard S, Legius E, Schwartz M, Matthijs G. Lack of Hardy-Weinberg equilibrium for the most prevalent PMM2 mutation in CDG-la (congenital disorders of glycosylation type la). Eur J Hum Genet. mai 2000;8(5):367-71.

5. Jaeken J, Stibler H, Hagberg B. The carbohydrate-deficient glycoprotein syndrome. A new inherited multisystemic disease with severe nervous system involvement. Acta Paediatr Scand Suppl. 1991;375:1-71.

6. PMM2 Congenital Disorder of Glycosylation [Internet]. Rare Diseases Clinical Research Network. [cité 17 août 2021]. Disponible sur: https://www.rarediseasesnetwork.org/fcdgc/pmm2

7. Matthijs G, Schollen E, Bjursell C, Erlandson A, Freeze H, Imtiaz F, et al. Mutations in PMM2 that cause congenital disorders of glycosylation, type la (CDG-la). Hum Mutat. nov 2000;16(5):386-94.

8. Stibler $\mathrm{H}$, Beaugé $F$, Bjørneboe A, Aufrère $\mathrm{G}$. Transferrin microheterogeneity in rats treated chronically with ethanol. Pharmacol Toxicol. avr 1989;64(4):383-5. 
9. Le Bizec C, Vuillaumier-Barrot S, Barnier A, Dupré T, Durand G, Seta N. A new insight into PMM2 mutations in the French population. Hum Mutat. mai 2005;25(5):504-5.

10. Matthijs G, Schollen E, Van Schaftingen E, Cassiman J-J, Jaeken J. Lack of Homozygotes for the Most Frequent Disease Allele in Carbohydrate-Deficient Glycoprotein Syndrome Type 1A. The American Journal of Human Genetics. mars 1998;62(3):542-50.

11. Matthijs G, Schollen E, Pardon E, Veiga-Da-Cunha M, Jaeken J, Cassiman J-J, et al. Mutations in PMM2, a phosphomannomutase gene on chromosome 16p13 in carbohydrate-deficient glycoprotein type I syndrome (Jaeken syndrome). Nat Genet. 1 mai 1997;16(1):88-92.

12. Pfeiffer JR, McAvoy BL, Fecteau RE, Deleault KM, Brooks SA. CARHSP1 is required for effective tumor necrosis factor alpha mRNA stabilization and localizes to processing bodies and exosomes. Mol Cell Biol. janv 2011;31(2):277-86.

\section{Figures}

\section{Figure 1.}

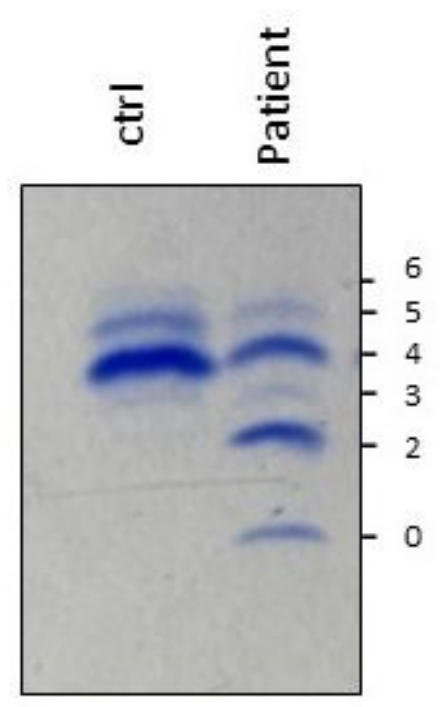

\section{Figure 1}

Distribution of transferrin glycoforms via transferrin isoelectrofocusing of the patient compared to a control

Numbers $0,2,3,4,5$, and 6 indicate the migration position of the asialo-, disialo-, trisialo-, tetrasialo-, pentasialo-, and hexasialotransferrin forms respectively. 
Figure 2.

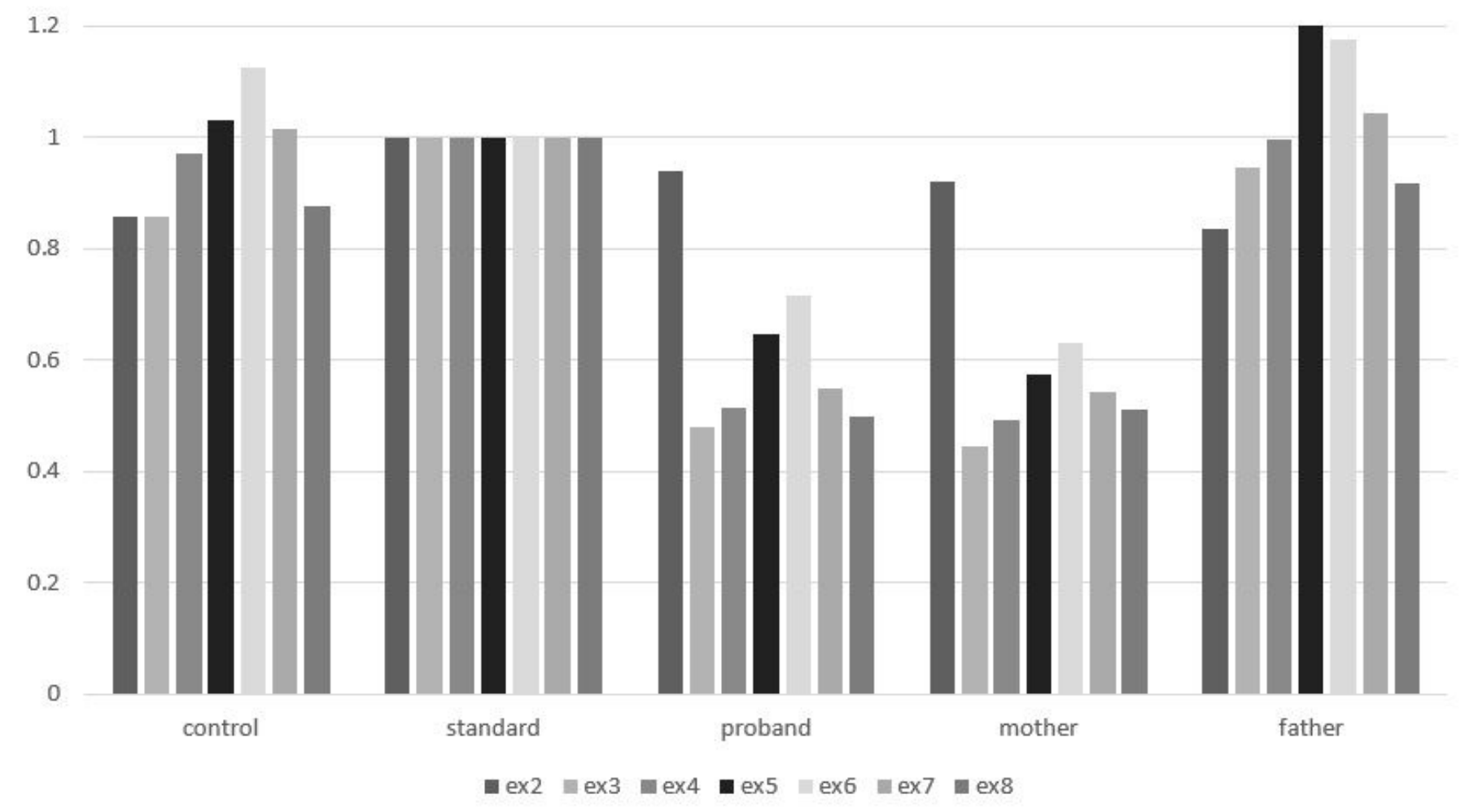

Figure 2

PCR quantification of PMM2 exons

qPCR of exons 2 to 8 of PMM2 in the proband and the parents compared to standard and control samples 


\section{Figure 3.}

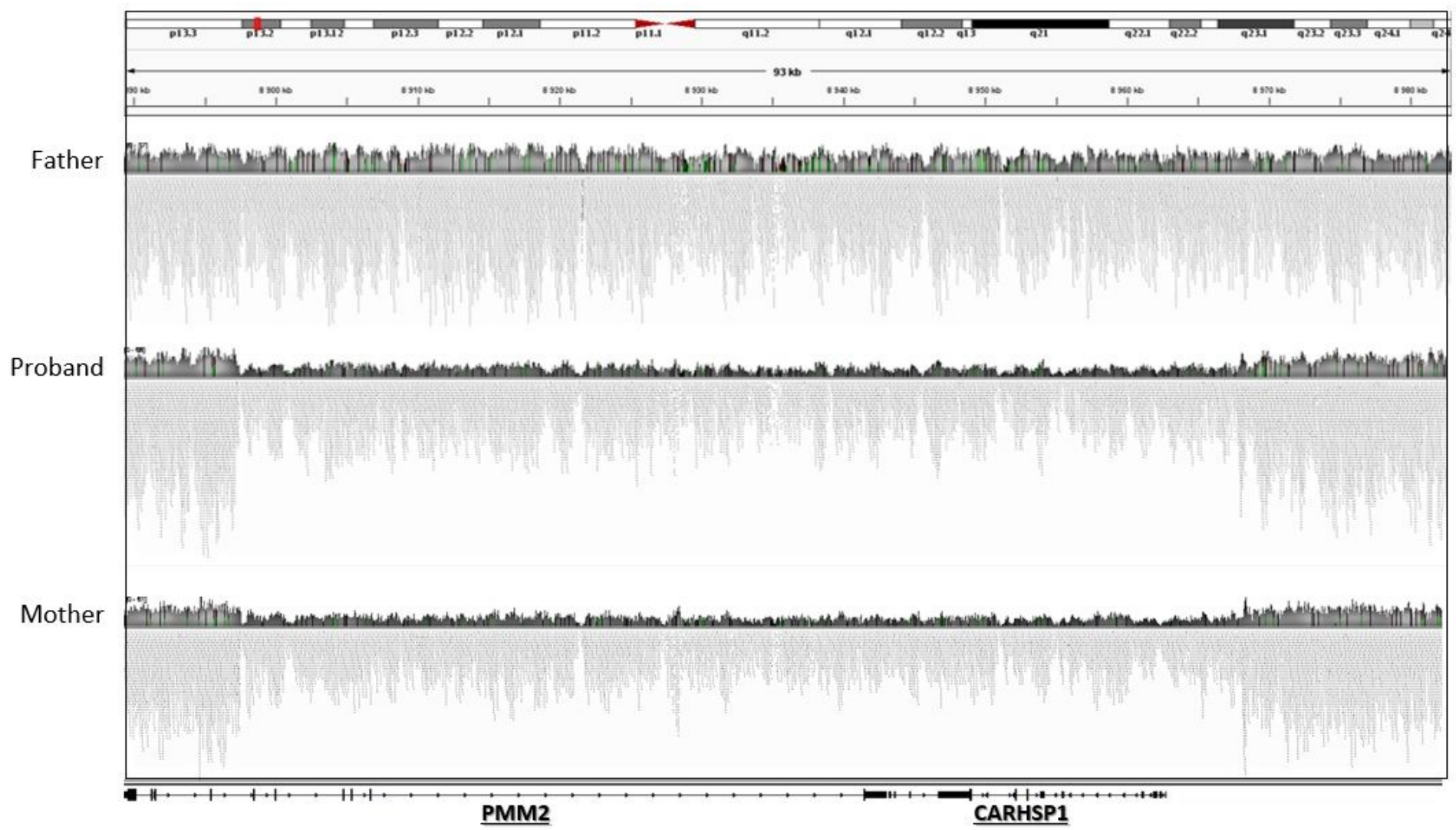

Figure 3

\section{Deletion breakpoint localisation}

Reads quantification in position chr16:8,897,826-8,968,278 of the family members. A deletion of $70453 \mathrm{pb}$ is seen for the proband and the mother. The genes affected by the deletion are named on the bottom of the figure. 